\title{
Research Opportunities in Space Propulsion
}

\author{
Stephen L. Rodgers \\ NASA/ Marshall Space Flight Center
}

\begin{abstract}
Rocket propulsion determines the primary characteristics of any space vehicle; how fast and far it can go, its lifetime, and its capabilities. It is the primary factor in safety and reliability and the biggest cost driver. The extremes of heat and pressure produced by propulsion systems push the limits of materials used for manufacturing. Space travel is very unforgiving with little room for errors, and so many things can go wrong with these very complex systems. So we have to plan for failure and that makes it costly. But what is more exciting than the roar of a rocket blasting into space?
\end{abstract}

By its nature the propulsion world is conservative. The stakes are so high at every launch, in terms of payload value or in human life, that to introduce new components to a working, qualified system is extremely difficult and costly. Every launch counts and no risks are tolerated, which leads to the space world's version of Catch-22:"You can't fly till you flown." The last big 'game changer' in propulsion was the use of liquid hydrogen as a fuel. No new breakthrough, low cost access to space system will be developed without new efficient propulsion systems. Because there is no large commercial market driving investment in propulsion, what propulsion research is done is sponsored by government funding agencies. A further difficulty in propulsion technology development is that there are so few new systems flying. There is little opportunity to evolve propulsion technologies and to update existing systems with results coming out of research as there is in, for example, the auto industry.

The biggest hurdle to space exploration is getting off the ground. The launch phase will consume most of the energy required for any foreseeable space exploration mission. The fundamental physical energy requirements of escaping earth's gravity make it difficult. It takes $60,000 \mathrm{~kJ}$ to put a kilogram into an escape orbit. The vast majority $(\sim 97 \%)$ of the energy produced by a launch vehicle is used to get propellants off the ground to be burned later. A modern launch vehicle is usually able to put no more than $1.5 \%-3 \%$ of its total liftoff weight into low earth orbit.

In-space propulsion systems provide the impulse for adjusting velocity, changing orbit altitude, controlling attitude, station keeping, and deorbiting at the end of a satellite's life. Rocket propulsion systems for long duration space missions will be required to operate reliably for long periods of time, withstand many work cycles and operate more efficiently. These propulsion needs are being satisfied currently by chemical propulsion and, increasingly, by electric propulsion subsystems. Propulsion systems for planetary exploration by large spacecraft currently don't exist. These systems, requiring large high power sources, long term storage of cryogenics, long life thrusters, and highly efficient propellant systems are all largely left to be developed and offer a wealth of research opportunities. It is worth noting that historically the bulk of funding for propulsion research and technology has gone to launch systems development and very little to in- 
space systems and virtually none to propulsion for planetary exploration. The physical requirements for in-space propulsion are also different than for launch propulsion in that due to the very low gravity environment the need for high thrust to weight propulsion systems are minimized as compared to launch propulsion. Consequently, as opposed to launch propulsion and earth focused satellite propulsion, there is potential for large performance gains for in-space thrusters, electric power generation and energy storage in space.

Propulsion relies on a multi-disciplinary collection of technologies (combustion, materials, fluid dynamics, chemistry, and mechanics for example) which makes it a challenging integration task. New developments in all or any of these technical disciplines can result in significant improvements in propulsion performance or reliability.

\section{Energetics}

Rocket propulsion can be divided into two types based on energy source: chemical and non-chemical. Most of the rocket propulsion systems flying today are chemical systems, which derive their energy from the combustion of propellants which produces high temperatures and pressures in the combustion chamber. These combustion products are expelled through a nozzle producing thrust. Propellants are used either in liquid or solid form and typically consist of the elements carbon, hydrogen, nitrogen, oxygen, and chlorine. These atoms need to be arranged into molecules that have the highest energy per unit molecular weight for maximum efficiency and performance. Huge payoffs can be had from increasing density or specific energy of propellants; unfortunately increasing energy density usually also means decreasing stability. Along with stability, considerations must be given to such characteristics as toxicity, cost, and ease of production. The propulsion world has reached something of a plateau in propellants that are actually used. While it may be difficult to beat hydrogen and oxygen for launch systems, opportunities exist in solid oxidizers and binders, monopropellants, and in-space propellants. Leading the need for new propellants will be environmental 'green' efforts and, long term, in-situ propellant production. Chemical propulsion will be with us for a long time, and will probably always be the method we use to get off the ground.

Non-chemical systems derive their energy from non-combusting, external energy sources. They typically have the advantage that, unlike chemical propellants which are limited to the energy they can carry in chemical bounds, non-chemical systems can achieve much higher temperatures and efficiencies by the addition of energy from an external source to the propellant. The only non-chemical propulsion system in operation today is electric propulsion in various forms. These systems operate with an external power supply, for example solar cells, and either heat a working fluid (as in thermal propulsion) or directly accelerate ions or plasma to produce thrust. These systems can reach higher velocities and propellant efficiencies but have much lower thrust levels Higher propellant efficiencies (or specific impulse) results in needing less propellant, and either decreasing spacecraft mass (less mass to launch) or increasing payload mass. It could also result in additional capability for satellite repositioning options or longer lifetime. Electric propulsion systems are increasingly being used in operational satellites today but it took approximately thirty years of research and development to fly the first 
operational system. This is a good example of the length of time it can take to get new propulsion technologies flying. Research directed at increasing the lifetime and decreasing the mass of the electric thrusters, power processing unit and feed systems will enhance the attractiveness of electric propulsion systems.

\section{Alternate Energetics}

Of the many advanced propulsion concepts for large scale space exploration, nuclear thermal propulsion (NTP) looks the most viable. Several efforts at developing nuclear based propulsion systems have been attempted in the past and so far, other than low power Radioisotope Thermoelectric Generators (RTG) based electric systems which have been very successful, we have yet to introduce a nuclear rocket into our inventory. Nuclear thermal propulsion saw a rather large developmental effort from the late 50's to the early 70's with the Rover program which progressed through feasibility design and demonstration tests of several nuclear rocket engine configurations. These encompassed reactors sized from $100 \mathrm{Mw}$, through 5,000 Mw. Cumulative testing at high power density for all Rover tests totaled 376 minutes. In an NTP design a nuclear reactor is used to heat hydrogen which is then expanded through a nozzle. This thermal propulsion concept is limited by the materials of the reactor and chamber and is typically limited to $2500-2700 \mathrm{~K}$. A nuclear rocket can achieve about twice the propellant efficiency of a typical chemical rocket but must carry the additional weight of the reactor and radiation shielding. Any nuclear propulsion system will also have to deal with the very real safety issues and regulatory burden. An alternative nuclear propulsion concept uses a nuclear reactor to power a thermal conversion power source, such as a Stirling engine, to produce electricity and drive an ion or plasma thruster. These type of space systems, along with the hardware associated with the nuclear device, also have to carry large space radiators to eliminate excess heat generated from the nuclear reactor, the power conversion unit, and the thruster.

Other sources of energy to drive advanced propulsion concepts have long been considered. These include other direct drive nuclear fission concepts, fusion, anti-matter and beamed energy. While these all are potentially attractive as propulsion energy sources, they all suffer from fundamental physics or scale-up issues and it is doubtful that a future propulsion potential will be sufficient to drive their exploration and development. Another class of space propulsion concepts are those that emit no propellant but instead works against their environments to achieve a change in velocity. An example is the electrodynamic tether wich generates an electrical field by moving within planetary electrodynamic fields. The resulting forces can be used to raise or lower the tether. Such a device, which carries no propellant or energy source on board may prove to have real niche applications.

Conventional propulsion systems are near peak performance levels. Further refinements in manufacturing, engineering design, and materials will provide increases in reliability and safety as well as lower costs. The next revolutionary step in propulsion will come from a breakthrough in energetics, either chemical or non-chemical. Propulsion fundamentally is a problem of energy storage density and energy to thrust conversion efficiency. 


\section{Materials}

Materials play a major role in determining the characteristics and performance of a rocket propulsion system. Performance enhancements can be obtained by either reducing the weight of the engine components or by providing extra temperature margin and a concomitant longer part life. Temperature is important but so is the ability to withstand thermal shock, cycling between cryogenic temperatures and high combustion temperatures, while maintaining durability and strength. The performance of thermal propulsion systems is limited by the temperature constraints of the combustion chamber, nozzle materials, and other elements exposed to high temperatures. Low cost, higher temperature materials and robust coatings are needed. Good candidates to achieve the higher temperatures desired are ceramics, intermetallics, and carbon-carbon compounds. The use of cryogenic propellants present specific material needs. Materials or coatings that are oxygen resistant for use in LOX environments and hydrogen embrittlement resistant materials for use with cryogenic hydrogen fuels are needed. Materials for lightweight insulations, LOX compatible light weight composite tanks and high temperature LOX resistant lines and chambers are also needed. Turbomachinery presents material opportunities as well. Material needs here include higher specific strength materials capable of withstanding the low temperature (liquid hydrogen) and high speed impellers environments, low cost net-shape fabrication of components, and wear and corrosion resistant bearings.

Material needs for solid motors include reduction in cost for $\mathrm{C}-\mathrm{C}$ processing, replacement of materials no longer widely available (for example rayon), higher temperature composite capabilities and oxidation resistance, reduced erosion nozzles, lighter weight insulations, advanced manufacturing processes (for example processes that allow the insulator and case to be wound on the cast propellant). In situ manufacturing diagnostics (NDE for example) is another area in materials research for solids which could reduce costs, increase reliability, and provide a means for monitoring a systems health during storage.

One of the biggest challenges in developing materials besides cost is the time frame it takes to fully develop a new material and make it available for use by the design engineers. Materials modeling to determine the correct chemistry, phase diagrams, material properties and optimize processing operations are another multi-disciplinary area which has large potential payoff; particularly for composites.

\section{Other Research Opportunities}

Models: The propulsion community has developed an extensive database of propulsion phenomena for many specific propulsion systems, but its a priori models still lag behind. If we get outside our databases we are in unknown territory. We still can't model accurately events such as combustion instability or two phase flow loss. Certainly the tools we have today are much better than they used to be, but for understanding phenomena or designing new hardware, they still need improvements. One reason for this is the difficulty in modeling such a highly integrated system. In order to adequately model these complex systems during all phases of operation it requires numerous complex models which have to communicate efficiently. The design of a propulsion 
system requires the use of a sophisticated set of design and analysis tools that must be able to communicate seamlessly. Engineering tools used for these complex systems are also required to meet rigorous verification and validation processes. In addition these systems are so complex that predictive models are generally fairly coarse. Most models are highly empirical and based on flight experience.

Integration: Propulsion systems are a complex system of hardware, combustion events, structures, dynamics, fluid flows, involving many technical disciplines from chemistry to various kinds of engineering. Research in propulsion is typically tackled one discipline at a time. Real progress could be made by working the 'system', considering how each component interacts or can assist another. Multifunction components, for example, could reduce mass and complexity. Integrating tanks and structure is a simple example. Others may be harder. Can the propellant function for radiation shielding? Can we consume the tank material structures as our propellant? Can we package the propellant into cartridges that can be loaded without ever exposing humans to toxic chemicals? Engineers like to work from requirements, but some creative thought in the beginning can deliver a more useful product.

Transition: One of the most difficult tasks for the researcher is to transition technology out of the laboratory to the users; this is especially true in propulsion. The small number of systems and the conservativeness prevalent in the space business makes it difficult to move ideas along the developmental path. Designers of new systems often use old components because of the lack of demonstrated performance and reliability of new components. This problem needs to be addressed by the funding agencies and the entire development pathway, including demonstrations, included in their roadmaps.

A favorite quote of mine comes from Theodore von Karman, "Those in charge of the future Air Forces should always remember that problems never have final or universal solutions, and only a constant inquisitive attitude towards science and a ceaseless and swift adaptation to new developments can maintain the security of this nation through world air supremacy." There perhaps is a tendency to think of propulsion as a mature technology, with just a little tweaking required. The truth is we have far to go to reach routine; and certainly as our goals change and as contributing technologies evolve, new propulsion technologies will arise. Our propulsion systems will only carry us as far as our research stretches.

Reading List

1. G.P. Sutton, Rocket Propulsion Elements, Sixth Edition, Wiley Interscience, New York, 1992

2. M.J.L. Turner, Rocket and Spacecraft Propulsion, Springer-Praxis, Chichester, UK, 2000

3. A. Whitaker, B. Cook, R.G. Clinton, Materials Requirements for Advanced Space Propulsion Systems, $\mathbf{4 3}^{\text {rd }}$ AIAA Aerospace Sciences Meeting, 10-13 January (2005), AIAA 2005-1441 
4. S.J. Scotti, C. Clay, M. Rezin, Environments Applied to Reusable Launch Vehicles, AIAA/ICAS International Air And Space Symposium and

Exposition: The Next 100 Years, 14-17 July, (2003), AIAA 2003-2697

5. R.H. Frisbee, Advanced Propulsion for the $21^{\text {st }}$ Century, Journal of Propulsion and Power, 19/6, (2003), 1129-1154

6. R.L. Forward, Alternate Propulsion Energy Sources, (1983), AFRPL-TR-83-067

7. J.A. Angelo, D. Buden; Space Nuclear Power, Orbit Book Company, Inc.; Malabar, FL, 1985 